\section{AB0331 PULMONARY INVOLVEMENT IN A SINGLE CENTER COHORT OF PATIENTS WITH SYSTEMIC LUPUS ERYTHEMATOSUS}

N. Senkal ${ }^{1}$, E. Kiyan ${ }^{2}$, E. Kocasoy-Orhan ${ }^{3}$, A. Demir ${ }^{4}$, M. Aydogan ${ }^{5}$, Y. Yalçınkaya ${ }^{6}$, A. Gul ${ }^{6}$, M. Inanc ${ }^{6}$, M. L. Ocal ${ }^{6}$, B. Artim-Esen ${ }^{6} .{ }^{1}$ Istanbul University Istanbul Faculty of Medicine, Internal Medicine, Istanbul, Turkey; ${ }^{2}$ Istanbul University Istanbul Faculty of Medicine, Chest Diseases, Istanbul, Turkey; ${ }^{3}$ Istanbul University Istanbul Faculty of Medicine, Neurology, Istanbul, Turkey; ${ }^{4}$ Fulya Radiologic Imaging Center, Radiology, Istanbul, Turkey; Istanbul University Istanbul Faculty of Medicine, Cardiology, Istanbul, Turkey; ${ }^{6}$ Istanbul University Istanbul Faculty of Medicine, Rheumatology, Istanbul, Turkey

Background: The prevalence of SLE pulmonary involvement varies depending on several factors, including diagnostic methods [1].

Objectives: We aimed to determine the frequency of involvement with different diagnostic methods in a single center cohort.

Methods: 300 SLE patients were included. Chest x-ray (CXR), lung spirometry, carbonmonoxide diffusion test (DLCOc) and echocardiography were performed. High resolution thorax computed tomography (HRCT) was done for a definite diagnosis of interstitial lung disease (ILD) whilst diagram electromyography (EMG), ultrasonography (USG) and magnetic resonance imaging (MR) were utilized to diagnose shrinking lung syndrome (SLS).

Results: The mean age and follow-up time were 43 and 11,5 years respectively. Of 300 patients, $16 \%$ had ILD, 6,7\% had pulmonary hypertension (PHT), 3\% had SLS, $0,3 \%$ had pulmonary infarction. At the start of the study, patients' records showed that $4 \%$ had ILD, $5 \%$ PHT, $0,3 \%$ SLS and $0,3 \%$ pulmonary infarction. The median age, mean duration of disease and follow-up time were significantly higher and longer in patients with ILD compared to patients without $(p<0.05)$. Forced expiratory volume (FEV1), forced vital capacity (FVC), DLCOc and total lung capacity (TLC) were significantly lower in patients with ILD and with SLS $(p<0,001)$. Patients with ILD had significantly higher frequency of arthritis, serositis, Raynaud myositis and anti-Scl70 positivity. Avascular necrosis, diabetes and malignancy were significantly more frequent in those patients. All patients with suspected SLS undergone diagram EMG, USG and MR. Out of 10 suspected cases, in 6 EMG, in 5 USG and in 9 MR was compatible with SLS diagnosis. 5 patients had 3 of the diagnostic methods positive to diagnose SLS. Muscle atrophy and weakness, avascular necrosis were more frequent in this group of patients $(p<0.05)$. There were more patients treated with mycophenolate mofetil (MMF) and cyclophosphamide in the SLS group whilst more with MMF in the ILD group. Significantly higher frequency of patients had stopped using hydroxychloroquine $(\mathrm{HCQ})$ in the ILD group $(\mathrm{p}=0,04)$

Conclusion: Interstitial lung disease is common in patients with SLE and considerable number of patients have SLS [2]. Spirometry, DLCOc and CXR are simple but valuable to diagnose pulmonary involvement in SLE patients. Diaphragm MR, USG and EMG are complementary methods for definite diagnosis in SLS [2]. Considering the significant difference of prevalence between the start and the end of the study, one of the possibbilities is the underrecognition of SLE pulmonary disease due to its being part of a multisystemic presentation. Higher usage of immunosuppressives in these patients may support a multisystemic active disease. Although drug effect is another concern, it is hard to establish a causal relationship due to the study's cross-sectional design. HCQ may have a role in ILD prevention. REFERENCES:

[1] Keane MP, Lynch JP. Pleuropulmonary manifestations of systemic lupus erythematosus. Thorax 2000;55:159-166.

[2] Singh R, Huang W, Menon Y, Espinoza LR. Shrinking lung syndrome in systemic lupus erythematosus and Sjogren's syndrome. J Clin Rheumatol. 2002 Dec;8(6):340-5

Table 1. Spirometry; DLCO; diaphragm EMG, USG and MRI results of patients with SLS.

\begin{tabular}{lcccccccccc}
\hline Patient/ & $1 /$ & $2 /$ & $3 /$ & $4 /$ & $5 /$ & $6 /$ & $7 /$ & $8 /$ & $9 /$ & $10 /$ \\
Age/ & $44 /$ & $57 /$ & $39 /$ & $38 /$ & $23 /$ & $60 /$ & $58 /$ & $37 /$ & $66 /$ & $28 /$ \\
Sex & $\mathrm{F}$ & $\mathrm{F}$ & $\mathrm{F}$ & $\mathrm{M}$ & $\mathrm{F}$ & $\mathrm{F}$ & $\mathrm{F}$ & $\mathrm{F}$ & $\mathrm{F}$ & $\mathrm{F}$ \\
\hline FEV1 (\%) & 47 & 65 & 59 & 63 & 67 & 62 & 71 & 53 & 70 & 39 \\
FVC (\%) & 56 & 73 & 59 & 62 & 79 & 70 & 72 & 55 & 62 & 37 \\
DLCO (\%) & 45 & 50 & 44 & 65 & 53 & 55 & 47 & 65 & 62 & 45 \\
TLC (\%) & 61 & 71 & 65 & 66 & 78 & 64 & 54 & 63 & 62 & 45 \\
USG deep inspiration (L) & 4,70 & 4,74 & 2,59 & 3,01 & 3,06 & 3,06 & 5,73 & 4,17 & 2,12 & 1,59 \\
USG deep inspiration (R) & 3,72 & 1,98 & 2,26 & 2,24 & 2,77 & 2,17 & 4,67 & 2,83 & 4,62 & 2,49 \\
USG diaphragm thickness (L) & 4,82 & 3,00 & 2,48 & 2,84 & 2,55 & 1,59 & 2,09 & 3,18 & 1,62 & 1,44 \\
USG diaphragm thickness R(R) & 1,23 & 1,04 & 1,84 & 1,80 & 2,19 & 1,31 & 1,79 & 1,97 & 2,08 & 1,63 \\
MR high side & $\mathrm{R}$ & $\mathrm{R}$ & $\mathrm{R}$ & $\mathrm{R}$ & $\mathrm{R}$ & & $\mathrm{R}$ & $\mathrm{R}$ & $\mathrm{L}$ & $\mathrm{L}$ \\
MR height difference & 4,92 & 6,19 & 2,87 & 2,64 & 1,67 & & 2,45 & 1,73 & 0,95 & 0,78 \\
EMG resting AMP (R) & 0,2 & 0,3 & 0,6 & 0,5 & 0,6 & 0,4 & 0,1 & 0,8 & 0,9 & 0,7 \\
EMG resting LAT (R) & 7 & 6,4 & 7,1 & 6 & 6,7 & 8 & 6,15 & 4,75 & 6 & 5,8 \\
EMG resting AMP (L) & 0,4 & 0,5 & 0,8 & 0,7 & 1,2 & 0,6 & 0,3 & 1,2 & 0,5 & 0,5 \\
EMG resting LAT (L) & 6,35 & 5,75 & 6,85 & 5,6 & 6,05 & 6 & 6 & 4,35 & 5,2 & 6,15 \\
& & & & & & & & & &
\end{tabular}

Disclosure of Interests: None declared

DOI: 10.1136/annrheumdis-2021-eular.3152

\begin{tabular}{|l|l|}
\hline AB0332 & IS MINOR SALIVARY GLAND HISTOLOGY A \\
GOOD BIOMARKER SCORE TO PREDICT THE \\
EXTRAGLANDULAR INVOLVEMENT IN PATIENTS WITH \\
PRIMARY SJOGREN'S SYNDROME?
\end{tabular}

A. Martins ${ }^{1}$, R. Ferreira ${ }^{1}$, S. Pimenta ${ }^{1,2}$, L. Costa ${ }^{1}{ }^{1}$ Centro Hospitalar Universitário São João, Rheumatology, Porto, Portugal; ${ }^{2}$ Faculdade de Medicina da Universidade do Porto - FMUP, Rheumatology, Porto, Portugal

Background: The minor salivary gland biopsy (MSGB) is considered the "gold standard" for the diagnosis of primary Sjögren's syndrome (pSS). The histological hallmark is focal lymphocytic infiltration with the presence of 50 or more lymphocytes per $4 \mathrm{~mm}^{2}$ (focus score $\geq 1$ ). Several studies have documented the association between focus score (FS) and extraglandular involvement, suggesting that FS could be used as a histological index of disease severity and a prognostic tool.

Objectives: To investigate the association between the FS and clinical, haematological and serological findings in a cohort of patients with pSS.

Methods: We performed a retrospective analysis of patients with pSS who fulfilled the ACR/EULAR 2016 classification criteria that were submitted to a minor salivary gland biopsy (MSGB). Demographic data, disease duration, extraglandular involvement and histopathologic data were collected and analyzed. The severity of histologic changes was graded according to the Chisholm and Mason scoring system and the FS was determined. Fisher's exact test/Chi-square test and Mann-Whitney $U$ test were used to compare the patients with and without positive biopsy ( $F S<1$ versus $F S \geq 1$ ). Futhermore, to investigate the association between the quantitative value of focus score in groups with and without each extraglandular manifestations the authors used the Mann-Whitney $U$ test. P-value $<0.05$ was considered statistically significant.

Results: A total of 59 pSS patients were included. Regarding the histopathologic pattern, 40 of 59 patients had a FS $\geq 1$, while 19 patients had a FS $<1$. Although described as having $F S \geq 1$, the pathologists failed to report the absolute value in 11 of $40(27,5 \%)$ patients. Germinal center-like structures were found in $1(1,7 \%)$ patient. The demographic and clinical features in pSS patients with $\mathrm{FS}<1$ and with FS $\geq 1$ are presented in table 1. Anti-SSA was strongly associated with FS $<1(p<0,001)$. No significant difference in demographic and clinical features were found between the two groups.

Table 1. Demographic and clinical features in pSS patients with FS $<1$ and with FS $\geq 1$ ( ${ }^{*}$ Fisher's exact/Chi-square test; † T-test; † Mann-Whitney U test).

\begin{tabular}{|c|c|c|c|}
\hline & FS $<1(n=19)$ & $F S \geq 1(n=48)$ & p-value \\
\hline Gender (female), n (\%) & $17(89,5)$ & $34(85,0)$ & 0,639 * \\
\hline Current age (years) (mean $\pm \mathrm{SD})$ & $60,11+-13,39$ & $54,78(+-13,48)$ & $0.16 \dagger$ \\
\hline Disease duration (years) (median, P25-P75) & $3(2-7)$ & $4(3-5)$ & $0,507 \ddagger$ \\
\hline \multicolumn{4}{|l|}{ Extraglandular involvement } \\
\hline Arthralgias/Arthritis, n (\%) & $6(31,7)$ & $22(55,0)$ & 0,092 * \\
\hline Myositis, n (\%) & $0(0)$ & $1(2,5)$ & - \\
\hline Pulmonary involvement, $\mathrm{n}(\%)$ & $3(15,8)$ & $5(12,5)$ & 0,704 * \\
\hline PNS involvement, $\mathrm{n}(\%)$ & $0(0)$ & $1(2,5)$ & - \\
\hline CNS involvement, $\mathrm{n}(\%)$ & $0(0)$ & $1(2,5)$ & - \\
\hline Cutaneous involvement, $\mathrm{n}(\%)$ & $1(5,3)$ & $1(2,5)$ & 0,544 * \\
\hline Lymphoma, n (\%) & $0(0)$ & $2(5,6)$ & - \\
\hline \multirow{2}{*}{\multicolumn{4}{|c|}{ Haematological and serological characteristics }} \\
\hline Leukocytopenia, n (\%) & & & \\
\hline Hypergammaglobulinaemia, n (\%) & $10(52,6)$ & $16(40,0)$ & 0,361 * \\
\hline RF positivity, $n(\%)$ & $5(26,3)$ & $10(25,6)$ & 1 * \\
\hline ANA positivity, n (\%) & $18(94,7)$ & $34(85,0)$ & $0,411^{*}$ \\
\hline Anti-SSA positivity, n (\%) & $19(100)$ & $21(52,5)$ & $<0,001$ * \\
\hline Anti-SSB positivity, n (\%) & $7(36,8)$ & $12(32,5)$ & 0,742 * \\
\hline ESSDAI score (median, P25-P75) & $2(0-8)$ & $2(0-7,25)$ & $0,816 \ddagger$ \\
\hline
\end{tabular}

When comparing the quantitative value of FS in groups with and without each extraglandular manifestation, the authors found that patients with articular involvement had a significant higher FS value than patients without articula manifestations $(p=0.037)$. No other significant association was found.

Conclusion: Regarding the clinical manifestations, we didn't find a significant difference between patients with a FS $<1$ and those with a FS $\geq 1$. Anti-SSA was strongly associated with a FS $<1$, which corroborates the importance of anti-SSA for the pSS diagnosis in patients with a negative biopsy. We found that a higher FS value was associated with articular involvement. However, this study has limitations such as a small sample and a low incidence of extraglandular manifestations. In conclusion, more studies are needed to clarify the associations found in the literature.

Disclosure of Interests: None declared

DOI: 10.1136/annrheumdis-2021-eular.3220 